\title{
Original manuscript by Yu et al.: Antibiotic prophylaxis in perioperative period of percutaneous nephrolithotomy: a systematic review and meta-analysis of comparative studies
}

\author{
Tuo Deng ${ }^{1} \cdot$ Guohua Zeng ${ }^{1}$ (1)
}

Received: 1 October 2019 / Accepted: 4 October 2019 / Published online: 28 October 2019

(c) Springer-Verlag GmbH Germany, part of Springer Nature 2019

\section{Dear Editor,}

With much interest, we read the meta-analysis by Yu et al. [1]. The authors performed a comprehensive review and pooled analysis to assess the effectiveness of different antibiotic prophylaxis strategies on preventing postoperative infection complications. Postoperative urinary tract infection leads to great pain and economic burden on patients, thus figuring out an appropriate strategy of antibiotic prophylaxis in percutaneous nephrolithotomy is necessary and important in clinical practice. In this aspect, Yu et al. did a good work. However, some methodology issues in their study should be addressed.

At first, the misuse of effect model in the meta-analysis should be pointed out. In the pooled analysis, the selection of effect model depends on the results of heterogeneity test, which is conducted through Chi square test based $Q$ - and $I^{2}$-statistic [2]. If no heterogeneity existed with a $P$ value $>0.10$, the fixed-effect model should be used. Otherwise, the random-effect model should be applied. In the Methods section of Yu et al.'s study, the use of effect model is described correctly. However, in the Results section, the use of effect model is confused. For example, in Figs. 5A and 6, the results of heterogeneity tests show all $P$ values are greater than 0.1 and $I^{2}<50 \%$, thus the fixed-effect model should be used; however, the authors use the random-effect model. The misuse of effect model will result in mistakes for the pooled results, and lead to wrong conclusion.

This comment refers to the article available at https://doi. org/10.1007/s00345-019-02967-5.

Guohua Zeng

gzgyzgh@vip.tom.com

1 Department of Urology and Guangdong Key Laboratory of Urology, The First Affiliated Hospital of Guangzhou Medical University, Kangda Road 1\#, Haizhu District, Guangzhou 510230, Guangdong, China
Another issue of Yu et al.'s study is the inconsistent use of pooled effect size: odds ratio (OR) and risk ratio (RR). Actually, the definitions and calculation formulas of OR and RR are different and should not be misused. In Yu et al.'s study, the types of all input data for generating forest plots are all the same. According to the Cochrane Handbook, when the input data types are tha same, the formula for calculating pooled effect size in forest plot is also the same within RevMan software [3]. Therefore, the pooled effect sizes in $\mathrm{Yu}$ et al.'s study should be consistent. However, the OR is used in Figs. 3 and 5, while the RR is used in Figs. 4 and 6. Thus, the authors should clearly clarify why they choose OR or RR as pooled effect size.

In conclusion, we should pay more attention to the methodological correctness when conducting a study; it will directly impact the reliability of the results and conclusions. Finally, we declare that we have no conflicts of interest.

\section{References}

1. Yu J, Guo B, Yu J, Chen T, Han X, Niu Q, Xu S, Guo Z, Shi Q, Peng X, Deng Z, Yang P (2019) Antibiotic prophylaxis in perioperative period of percutaneous nephrolithotomy: a systematic review and meta-analysis. World J Urol. https://doi.org/10.1007/ s00345-019-02967-5

2. Higgins JP, Thompson SG, Deeks JJ, Altman DG (2003) Measuring inconsistency in meta-analyses. BMJ 327:557-560

3. Higgins JPT, Green S (eds) (2011) Cochrane Handbook for Systematic Reviews of Interventions Version 5.1.0 [updated March 2011]. The Cochrane Collaboration. http://www.cochrane-handb ook.org

Publisher's Note Springer Nature remains neutral with regard to jurisdictional claims in published maps and institutional affiliations. 